\title{
EL ESTADO DE BIENESTAR:
}

\section{UNA APROXIMACIÓN A LA INTERVENCIÓN ESTATAL}

\author{
Autor: León Julio Arango Buelvas ${ }^{1}$
}

\section{RESUMEN}

1 stado de Bienestar es el que pretende que los gobiernos les suministren a sus ciudadanos más necesitados, desde la cuna hasta la sepultura: Educación, vivienda, salud, pensiones de jubilación, empleo en la burocracia oficial, subsidios de desempleo, de invalidez... transporte público módico, cuidado de los niños, reparación de las víctimas, atención de los desplazados... (Hernán 'Gonzáles Rodríguez). Normalmente se relaciona con el componente social de las economía lo que implica tomar medidas ante la existencia de problemas que perturban el dinamismo de las economías, por lo que se requiere la actuación del Estado a través de instrumentos y de mecanismos jurídicos que a nivel institucional se dirigen a resolver dichos problemas, independientemente a que estos sean de tipo estructural o coyuntural. Está orientado a dinamizar medidas favorables a cierto grupo de la sociedad que por efecto del mercado y de los intereses que persigue, tiene limitaciones para alcanzar posiciones diferentes. Desde el punto de vista de las diferencias sobre el Estado de bienestar se concluye que las posiciones a favor o en contra son de tipo ideológico, dada la dificultad de cuantificar algunas posiciones.

\section{Palabras claves: Estado de Bienestar, Componente Social, Gobierno.}

\section{Abstract}

Welfare State is the one that wants governments to provide their most needy citizens, from the cradle to the grave: Education, housing, health, retirement pensions, employment in the official bureaucracy, unemployment benefits, disability ... Modest public transportation, child care, reparation of victims, care of the displaced ... (Hernán'Gonzáles Rodríguez). Normally, it is related to the social component of the economy, which implies taking action against the existence of problems that disturb the dynamism of the economies, for which reason the State's action is required through instruments and legal mechanisms that at institutional level Address these problems, regardless of whether they are structural or cyclical. It is aimed at boosting measures favorable to a certain group of society that, due to the effect of the market and the interests it pursues, has limitations to reach different positions. From the point of view of differences on the welfare state we conclude that the positions for or against are ideological, given the difficulty of quantifying some positions.

Key words: Welfare State, Social Component, Government.

1. Docente Universidad de Sucre.- Economista. 


\section{EL ESTADO DE BIENESTAR: UNA APROXIMACIÓN A LA INTERVENCIÓN ESTATAL}

\section{INTRODUCCIÓN}

I Estado de bienestar normalmente se relaciona con el componente social de las economía lo que implica tomar medidas ante la existencia de problemas que perturban el dinamismo de las economías, por lo que se requiere la actuación del Estado a través de instrumentos y de mecanismos jurídicos que a nivel institucional se dirigen a resolver dichos problemas, independientemente a que estos sean de tipo estructural o coyuntural.

Cabe anotar que ante una presunta inexistencia de problemas no se puede inferir la ausencia de los lineamientos estatales debido a que la presencia institucional pública es fundamental para el direccionamiento de las actividades que afecten los objetivos propuestos.

En términos de aplicación de políticas es normal considerar que un gobierno puede actuar de varias formas, dentro de las cuales puede ser agente activo en la economía, regulador o cumpliendo funciones de supervisor. Lo anterior puede darse, sin alejarse del papel que le corresponde alrededor del bienestar social y orientando siempre sus acciones hacia la solución de los problemas o situaciones presentadas.

Teniendo en cuenta este planteamiento, hay que tener claro que la política pública debe actuar en un escenario o campo de acción para su operacionalización y hacerla efectiva en función de los objetivos que se busca con su implementación. Es decir debe tener un direccionamiento en el campo económico, social, ambiental, internacional o de otra índole 


\section{EL ESTADO DE BIENESTAR: UNA APROXIMACIÓN A LA INTERVENCIÓN ESTATAL}

\section{GENERALIDADES DEL ESTADO DE BIENESTAR}

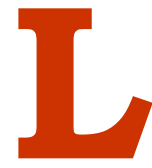

a búsqueda de un Estado de bienestar implica establecer condiciones para el desarrollo de muchas actividades no solo económicas sino sociales, políticas y ambientales, entre otras, por parte del Estado, o en muchas ocasiones cuando éste asigne responsabilidad al sector privado.

Este proceso conlleva a determinar el grado o nivel de intervención del Estado en los asuntos a tratar, considerando que existen niveles altos, medios y bajos de intervención estatal, empezando con ello el diseño de medidas para alcanzar objetivos específicos. Estas medidas se traducen en políticas públicas que deben estar dirigidas a la solución de problemas a la sociedad, tratando de consolidar de paso un Estado de bienestar.

De acuerdo con Stiglitz (2000, p. 17) existen cuatro causas o situaciones que pueden determinar la incapacidad sistemática del Estado para cumplir con los objetivos propuestos:

Información limitada. Indica con esto que muchas medidas tienen consecuencias complejas y difíciles de prever y muchas veces los gobiernos no disponen de la información necesaria para tomar decisiones.

Control limitado de las empresas privadas. Bajo esta causa el Estado no controla totalmente las consecuencias de sus intervenciones.

Control limitado de la burocracia. Se llega a esta causa porque el Congreso aprueba las leyes y delega su ejecución en otro organismo público. Este organismo puede tardar mucho en redactar la reglamentación correspondiente, cuyo contenido es fundamental para determinar las consecuencias de la legislación. En muchos casos, el hecho de que no se lleven a cabo los propósitos del Congreso no es algo intencionado sino una consecuencia de la ambigüedad de sus intenciones. En otros casos, los problemas se deben a que los burócratas carecen de los incentivos necesarios para poner en práctica la voluntad del Congreso.

Limitaciones impuestas por los procesos políticos. Se indica con esto que aunque los gobiernos estuvieran perfectamente informados de las consecuencias de todas las distintas medidas posibles, el proceso político a través del cual se toman las decisiones plantearía otras dificultades.

Siendo la sociedad la receptora de las acciones del Estado de bienestar, "el nuevo contexto político y económico internacional, así como las exigencias nacionales, regionales y locales para aumentar la democracia, la participación, la autonomía y para fortalecer, por ejemplo, las medidas para la protección del medio ambiente, ponen a los Estados frente a nuevas problemáticas" (Roth 2009, p. 17). Agrega Roth (2009) que esta nueva agenda política, que se adiciona a los habituales problemas de las políticas económicas y sociales de inequidad, pobreza creciente, corrupción, violencia e inseguridad, influye sobre el funcionamiento del Estado.

Al no poder deslindar al ente gubernamental en el establecimiento del Estado de bienestar es fundamental considerar el papel del Estado en este proceso, que en ocasiones conlleva a la universalización de muchos servicios y actividades. 


\section{EL ESTADO DE BIENESTAR: UNA APROXIMACIÓN A LA INTERVENCIÓN ESTATAL}

\section{GENERALIDADES DEL ESTADO DE BIENESTAR}

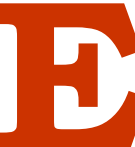

$s$ decir, el Estado como determinante de la prestación de servicios educativos, de saneamiento básico, manejo de subsidios, garantizar seguridad social, propiciar condiciones adecuadas para el mercado laboral, entre otras.

Dado el contexto y la estructura de las economías, así es el papel del Estado en función del bienestar por la relación que existe entre dicha estructura y la política a utilizar toda vez que "parece obvio que las políticas sociales pueden explicarse a partir de la estructura social, y que a su vez producen unos determinados impactos sobre esa misma estructura social" (Adelantado 2000, p. 23).

\section{LAS EXTERNALIDADES COMO EJEMPLO DE INTERVENCIÓN}

Una de las formas de expresión de la visualización del papel del Estado en función de la sociedad es a través de la presencia de externalidades, que es resultado del proceso de realización de las actividades económicas de los entes encargados de llevarlas a cabo y mediante las cuales necesariamente tienen una relación directa con el medio y con la sociedad, dados los objetivos que tienen como organización y los intereses que persiguen en función de sus actividades.

Amparados en su formación empresarial, constituida legalmente, y en el derecho de propiedad que les otorga la normatividad respectiva, se legaliza y legitima el accionar de los entes económicos, quienes desarrollan y dinamizan sus procesos productivos que en muchos ca- sos tienen consecuencias económicas, sociales y ambientales para las regiones, y todo en cumplimiento del objetivo de las organizaciones. Estas consecuencias son conocidas como Externalidades.

Cabe anotar que con la presencia de los efectos de las organizaciones en el medio, se acude al Estado para que en cumplimiento del objetivo benefactor, en función de la sociedad, intervenga en la solución de las consecuencias que son producto del interés organizacional.

En términos generales conocemos las externalidades como el efecto que genera en la sociedad o en el medio la acción de un agente económico. Es decir que una acción implica o da como resultado una consecuencia que puede tener connotaciones económicas, sociales o ambientales, entre otras. Esta relación de causa efecto debe ser controlada por los mismos agentes económicos o mediante la intervención del Estado, que en última instancia termina siendo el responsable de garantizar las condiciones de bienestar de la sociedad.

Ahora bien, dado el proceso de crecimiento de las economías y la búsqueda de condiciones diferentes a las naturales, se actúa en función de un mejor bienestar. De ahí la acción del hombre en torno a la producción económica y la reproducción del capital. Para Luxemburg (1985, p. 60) el capital necesita que todas las capas y sociedades no capitalistas se conviertan en compradores de mercancías y que le vendan sus productos. 


\section{EL ESTADO DE BIENESTAR: UNA APROXIMACIÓN A LA INTERVENCIÓN ESTATAL}

\section{GENERALIDADES DEL ESTADO DE BIENESTAR}

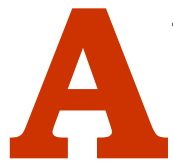

firma que parece que aquí comenzase la paz, la igualdad, la reciprocidad de los intereses, la competencia pacífica y los influjos civilizadores. Este planteamiento de Luxemburg determina un nivel específico de intervención estatal, que puede ser alto o bajo, dependiendo del efecto causado por los agentes del proceso.

En otro orden de ideas Mishan (1983, p. 159) plantea que "con el incontrolado deterioro del medio ambiente en las ciudades, pueblos y suburbios, la gente que huye tiene cada vez más sitios de los cuales huir". Dentro de las actividades económicas destructoras considera que el turismo va a la vanguardia de la destrucción por el proceso de transformación que lleva implícito tal actividad. Esta otra forma de expresión del efecto económico en la sociedad en muchas ocasiones pasa desapercibida por el impacto o la ilusión de los individuos ante el nuevo panorama de la economía.

En el proceso de la corrección de los problemas surgidos en desarrollo de las actividades económicas, y concretamente en el caso de una externalidad negativa como la contaminación, Miller (2002, p. 763) sostiene que quizá no sea apropiado fijar un impuesto uniforme de acuerdo con las cantidades físicas de contaminación. Considera que se debe establecer la cantidad de daños económicos, en lugar de tomar la cantidad de contaminación. Sobre el particular anota que:

Si se estableciera una forma de tributación para alinear los costos privados con los costos sociales y obligar a que las personas internalicen las externalidades, de alguna manera se llegaría a una medición de costos económicos en lugar de cantidades físicas. No obstante, el impuesto en cualquier evento, recaería sobre el sector privado y modificaría el comportamiento de los agentes económicos. Por tanto, debido a que el costo económico por la misma cantidad de contaminación sería distinto en diferentes localidades de acuerdo con la densidad de la población, la formación natural de las montañas, ríos, etc., los impuestos óptimos por la contaminación variarían de un punto geográfico a otro. La literatura económica plantea la presencia de un mercado competitivo como el idealismo del sistema al que denomina competencia perfecta, cuya existencia está en función de unos supuestos, siendo el eje central de este mercado la eficiencia en la asignación de los recursos. Esta eficiencia es fundamental para el funcionamiento de la economía del bienestar.

Como en el mundo económico siempre se parte del equilibrio de los mercados y siendo éstos ineficientes en presencia de externalidades, las situaciones presentadas conllevan a explorar algunas indicaciones para la asignación de los recursos mediante diferentes mecanismos cuyos resultados sean eficientes, por lo tanto "lograr una asignación eficiente en presencia de externalidades significa esencialmente asegurarse de que los agentes pagan el precio correcto por sus acciones" (Varian 2000, p. 507).

Como solución a los problemas generados por externalidades, Varian (2000, p. 508-510) destaca el papel de la política fiscal a través de los impuestos pigouvianos. 


\section{EL ESTADO DE BIENESTAR: UNA APROXIMACIÓN A LA INTERVENCIÓN ESTATAL}

\section{GENERALIDADES DEL ESTADO DE BIENESTAR}

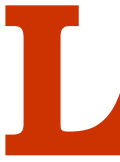

os impuestos pigouvianos son impuestos correctivos. Sobre estos plantea que lo que ocurre simplemente es que el precio al que se enfrenta la empresa por su acción no es el adecuado, por lo que puede obligársele a pagar un impuesto correctivo que asigne eficientemente los recursos. Agrega que el problema de esta solución se halla en que las autoridades fiscales deben conocer los costos de la externalidad.

Ahora bien, dada la relación del entorno social con los bienes considerados públicos, se debe resaltar que es fundamental la participación del recurso estatal en la provisión de dichos bienes. Al respecto afirma Benegas (1998, p. 205), que de ese modo se continua diciendo, los beneficiarios de externalidades positivas financian el producto vía impuestos, no existiendo, por ello, free-riders, lo que conlleva a desaparecer la falla de mercado. Esta última lo asimila a la producción de externalidades no internalizables.

De ello se deduce el papel del Estado en términos de la satisfacción social de necesidades y el suministro óptimo de bienes públicos, en contraposición al papel que jugaría en esta situación el sector privado dados sus intereses individuales, diferenciándose aquí las externalidades internalizables de las no internalizables.

\section{POSICIONES IDEOLOGICAS FRENTE AL ESTADO DE BIENESTAR}

A raíz de los interés de los participantes en las actividades económicas es normal encontrar posiciones contrarias alrededor del Estado de bienestar, es decir argumentos a favor y en contra de dicho Estado. Al respecto Sachs (2008, p. 341) sostiene que por efectos de la globalización los ideólogos de la libertad de mercado advierten que la competitividad se ha vuelto más intensa, lo que determina que debe concentrarse la atención en la competitividad y en el crecimiento económico por la amenaza exterior a la prosperidad de un país, por lo tanto se deben eliminar los obstáculos para el desarrollo de las empresas, al igual que para el ahorro y la inversión. Esto conlleva a disminuir impuestos y favorecer la obtención de beneficios porque la posición de un país en la economía mundial se verá afectada si tiene que soportar la carga de un gasto social elevado. Mientras que los defensores de la inversión social sostiene que se debe elevar mucho el gasto social precisamente porque la globalización desgarra el tejido de igualdad económica.

En el mismo sentido agrega Sachs (2008, p. 342) que para los opositores al Estado de bienestar una red de prestaciones sociales ralentizaría la sustitución de los sectores atrasados por otros emergentes y frustraría los beneficios de la iniciativa empresarial y la innovación. La opinión contraria sostiene que por las turbulencias del capitalismo es de vital importancia tender una red de prestaciones sociales que recabe el apoyo de la población a una economía sometida a fluctuaciones constantes. 


\section{EL ESTADO DE BIENESTAR: UNA APROXIMACIÓN A LA INTERVENCIÓN ESTATAL}

\section{GENERALIDADES DEL ESTADO DE BIENESTAR}

.Otra de las diferencias planteadas es que los primeros consideran el gasto social como una amenaza para la eficiencia económica y para las libertades personales, por lo que se debería dejar que los mercados ofrecieran por sí solo la mayor parte de los servicios que presta el Estado de bienestar. Por su parte la opinión contraria es que una red de prestaciones sociales garantiza la confianza en el futuro, posibilita que las personas asuman riesgos y favorece una redistribución de la riqueza que impide la desigualdad económica más extrema. Agregan que cuando no existe una red de prestaciones sociales sustentada por el gobierno, se deja que los más pobres se las arreglen por si solos y son condenados a vivir en la miseria, porque "solo los gobiernos ofrecen prestaciones de desempleo o ayudas cuando se producen catástrofes naturales importantes. Es ingenuo suponer que los mercados ofrecerán la protección conveniente a quienes la necesitan en unas circunstancias tan singulares y calamitosas" (Sachs 2008, p. 344) 


\section{EL ESTADO DE BIENESTAR: UNA APROXIMACIÓN A LA INTERVENCIÓN ESTATAL}

\section{CONCLUSIONES}

$\mathbf{E}$

I Estado de bienestar es la forma de expresión de la intervención estatal, la cual no está ausente en ningún sistema, pero el grado de intervención está en función de las necesidades y requerimientos del mismo sistema, de ahí que se encuentran nivel altos, medios o bajos de intervención.

Al cumplir funciones y desempeñar el papel estatal en muchas ocasiones se puede pensar en la universalización de servicios por parte del Estado, sin embargo para alcanzar los objetivos propuestos circunstancialmente existen causas que dificultan el proceso, lo que determina la incapacidad de alcanzar lo propuesto. Tales causas corresponden a la limitación de la información, control limitado de las empresas privadas, control limitado de la burocracia y las limitaciones que se imponen a través del proceso político.

El Estado de bienestar está orientado a dinamizar medidas favorables a cierto grupo de la sociedad que por efecto del mercado y de los intereses que persigue, tiene limitaciones para alcanzar posiciones diferentes. En este campo se sitúa el mercado laboral, la educación, manejo de subsidios, entre otros. Pero también hay acciones del Estado de bienestar orientadas a corregir las externalidades que corresponden a fallos del mercado.

Desde el punto de vista de las diferencias sobre el Estado de bienestar se concluye que las posiciones a favor o en contra son de tipo ideológico, dada la dificultad de cuantificar algunas posiciones. 


\section{EL ESTADO DE BIENESTAR: UNA APROXIMACIÓN A LA INTERVENCIÓN ESTATAL}

\section{BIBLIOGRAFÍA}

Adelantado J. (2000). Cambios en el Estado de Bienestar. Barcelona, Icaria Editorial S, A. Recu perado de: http://books.google.com.co/books

id=tP39vFG56S0C\&printsec=frontcover\&dq=el+estado+de+bienestar\&hl=es

419\&sa=X\&ei=X54IU4_WOo7LkQew2oDADg\&ved=0CCsQ6AEwAA\#v=onepage\&q=el 20estado\%20de\%20bienestar\&f=false

Luxemburg, R. (1985). La acumulación del capital, volumen II. Barcelona: Editorial Orbis S.A,.

Miller, Roger Leroy 2002. Economía Hoy. Colombia Pearson.

Mishan, E. J. (1983). Los costes del desarrollo económico. Barcelona: Editorial Orbis S.A.

Roth, A. (2009). Políticas públicas: Formulación, implementación y evaluación. Bogotá, Ediciones Aurora.

Sachs, J. (2008). Economía para un planeta abarrotado. Venezuela, Debate editores.

Stiglitz J. (2000). La economía del sector público. España, Antoni Bosch Editor.

Varian, Hal. (2002). Análisis microeconómico. Barcelona: Antoni Bosch Editor. 